$6^{\text {th }}$ Theoretical Fluid Mechanics Conference, June 27-30, 2011, Honolulu, HI

\title{
The scenario of two-dimensional instabilities of the cylinder wake under EHD forcing: A linear stability analysis
}

\author{
Juan D’Adamo ${ }^{\ddagger}$, Leo M. González ${ }^{\dagger}$, Alejandro Gronskis ${ }^{\ddagger}$, and Guillermo Artana ${ }^{\ddagger}$ \\ ${ }^{\dagger}$ Canal de Ensayos Hidrodinámicos, School of Naval Arquitecture, Universidad Politécnica de Madrid, Spain \\ ${ }^{\ddagger}$ Laboratorio de Fluidodinámica. Facultad de Ingeniería. Universidad de Buenos Aires, Argentina
}

\begin{abstract}
We propose to study the stability properties of a wake air flow forced by electrohydrodynamic (EHD) actuators, dielectric barrier discharge (DBD) type. EHD actuators can add momentum to the flow around an object in regions close to the wall. As the forcing frequencies, characteristic of DBD, are much higher than the natural shedding frequency of the flow ( $\mathrm{kHz} v \mathrm{vs}$. Hz), we consider in this work the forcing actuation as stationary. The actuators are disposed symmetrically near the boundary layer separation point.

We study the flow around a cylinder modified by EHD actuators experimentally by means of particle image velocimetry (PIV) measures and also computationally by solving the generalized eigenvalue problem obtained from linearized Navier-Stokes equations. In the numerical part, the presence of the EHD actuators has been implemented as a boundary condition on the cylinder surface. The base flow obtained computationally using this boundary condition has been compared with the experimental one in order to match the control parameters from both methodologies. Once the base flow agreement has been verified, the Hopf bifurcation produced when the flow starts the vortex shedding has been studied by different methods: first, when the baseflow is obtained from experimental snapshots, the evolution of the amplitude velocity oscillations is monitored; second, when the baseflow is computationally obtained, a global linearized eigenvalue problem is solved for the instability study. Finally, the critical parameters have been compared.
\end{abstract}

\section{Introduction.}

The simply geometry and the complex behavior that exhibits the flow around a cylinder represents, for low Reynolds numbers $\left(R e=U_{0} D / \nu<190\right)$, a prototypical 2D wake flow. The regime is identified with the Bénard von Kármán (BvK) vortex street, when the flow breaks its time continuous invariance at the bifurcation $R e=48.5$. In this case, the velocity field in the whole flow domain oscillates with the same global frequency and its harmonics, related to the appearance of a sufficiently large region of absolute instability in the near wake. As the oscillation amplitudes depend on the monitored position, the area that contains non zero oscillation amplitude points defines an instability region. Previous studies Zielinska et al. ${ }^{1}$ and Wesfreid et al. (1996), ${ }^{2}$ characterized the behavior of this region determining scale laws for its nonlinear evolution as functions of the Reynolds number.

The flow features can be altered by forcing parameters that may be varied smoothly and accurately to give rise different regimes. In this sense, we study this benchmark in order to characterize flow control in wakes by means of electrohydrodynamical actuators (EHD). EHD actuators for flow control have been receiving special attention in the last years, as reviewed by Moreau (2007). ${ }^{3}$ Among all the types of low-energy plasma actuators, one can notice a group of actuators that produces surface discharges. With these devices the goal is usually to use an electric wind produced by the plasma in order to modify the properties of the boundary layer close to the wall. A particular type of discharge is the surface dielectric barrier discharge (DBD). This 
discharge has been perfected for the first time in air at atmospheric pressure by Masuda $(1979)^{4}$ for ionic charging of particles. Roth (1998) et al. used it for airflow applications at the end of the 1990s, ${ }^{5}$ characterizing the momentum injected for flat plate and airfoil flows. Later, Thomas et al. $(2006)^{6}$ applied the DBD for the bluff body flow control. In their results, the authors showed drastic reductions of the flow separation and the associated Karman vortex shedding. On the other hand, some hypothesis on the dynamics of the DBD forcing arose from a computational study validated with PIV experiments in Gonzalez et al.(2009). ${ }^{7}$ From a stability analysis for a bluff body flow, with no vortex shedding, the forcing is introduced numerically as a moving surface boundary condition. In this line, the work by Gronskis $(2009)^{8}$ analyzed numerical models for DBD forced flows for a cylinder at low Reynolds numbers $(R e<200)$.

We intend with this work to optimize the DBD control device by analyzing the stability properties of the flow. For this reason, we study the possibility of suppressing the vortex sheading when a DBD actuator controls the flow. The work has been carried out from both experimental and computational points of view. Global modes evolves as the mean flow is modified by external parameters, Barkley $(2006)^{9}$ introduced hypothesis to consider the mean flow as a base flow for 2D stability analysis. In this way, the mean flow represents a marginal stability state and defines the frequency and amplitude of the wake oscillations. The nonlinear saturation of the oscillatory instability is achieved by Reynolds stresses from the mean flow modification. Khor et al. $(2008)^{10}$ and Leontini et al. $(2010)^{11}$ support these hypothesis from experimental and numerical data for Re up to 600 . These ideas have been applied to analyze forced wakes. Previous studies on the stability properties of forced wakes, Thiria and Wesfreid $(2007)^{12}(2009),{ }^{13}$ confirmed that a nonlinear critical behavior takes place under forcing. Indeed, a bifurcation scenario reappears as the forcing action stabilizes the wake fluctuations, in their case, a rotary oscillation. Figure 1, adapted from, ${ }^{12}$ shows how the global modes develop under forcing. The control actuation is represented by a vertical line for $R e>R e_{c}$, that varies the reverse flow region when it is present.

An interesting interpretation of the influence of a generic slip boundary condition on the wake dynamics was done by Legendre in, ${ }^{14}$ where shedding transition is interpreted as a critical accumulation of surface vorticity. In this context, we pursue the characterization of the DBD control actuator to complete an understanding which will eventually lead to a more effective energy consumption. We organize the work as it follows: in section II we describe the experimental setup to produce the DBD discharge as well as the method to measure the velocity fields of the flow, in section III we detail the characteristics of the problem, boundary conditions and baseflow properties, in section IV the numerical methodology to perform the linear global is described, in section $\mathrm{V}$ the results obtained in both approaches are shown and compared, at last we resume some conclusions and formulate perspectives for future works.

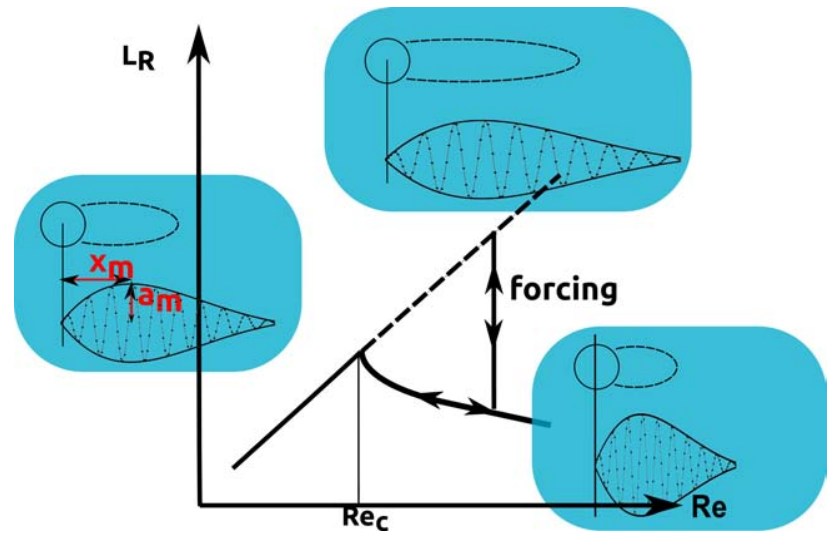

Figure 1. The reverse flow region $L_{R}$ behind the cylinder evolves nonlinear with $R e$, as it is modified by the mean flow. A new bifurcation scenario appears under forcing conditions.

\section{Experimental setup.}

The experimental set-up consists of a flow around a circular cylinder at low Reynolds number, $R e \sim 200$. The velocity field measurements were undertaken with the cylinder placed in a closed loop wind tunnel with 
a test section of $50 \times 50 \mathrm{~cm}^{2}$, where the air velocity is $U o \simeq 0.17 \mathrm{~m} / \mathrm{s}$.

The EHD actuator was mounted on the surface of a polymethyl methacrylate cylinder tube with external diameter $D=20 \mathrm{~mm}$. The tube wall was $2 \mathrm{~mm}$ thick.

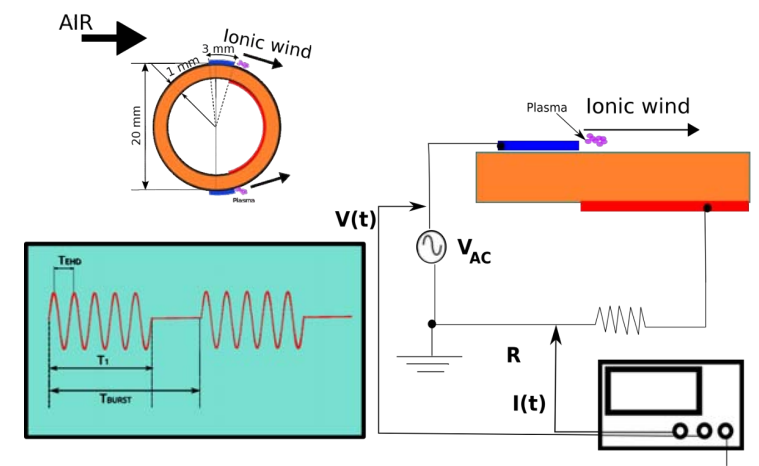

Figure 2. Schematic of the EHD actuator: Electrodes disposed flush-mounted. Electric circuit and input signal detail.

The electric circuit is composed of a signal generator, an audio amplifier and an ignition coil to give a discharge at $V_{E H D}=10 \mathrm{kV}$ and a frequency of $f_{E H D}=5.6 \mathrm{kHz}$. The signal amplitude is then modulated by bursting the signal as showed by Figure 2. A second frequency arises $f_{\text {Burst }}=1 / T_{\text {Burst }}$ and the electrical energy delivered by the device is characterized by a duty cycle $\left(D C=T_{1} / T_{B u r s t}\right)$. Special care was taken to assure a stationary input as $f_{\text {Burst }} \gg f_{\text {flow }}$, being $f_{\text {flow }}$ the vortex shedding frequency $(\simeq 1.5 \mathrm{~Hz}$, as the Strouhal number $S t=f D / U_{0} \simeq 0.2$ ). In short, the flow control parameter is the Duty Cycle (DC) input that modulates the "ionic wind" momentum.

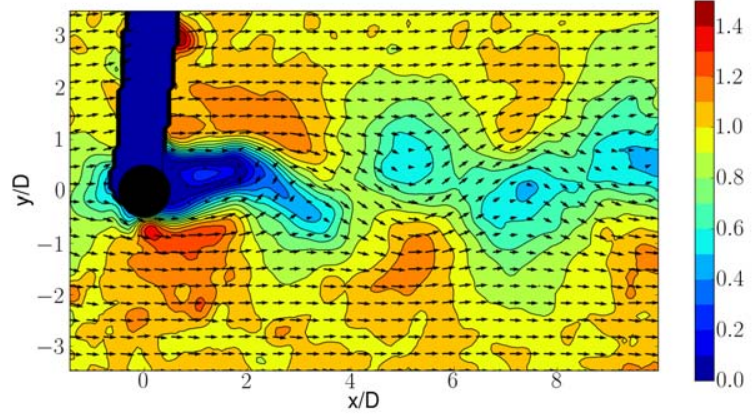

(a) No forcing.

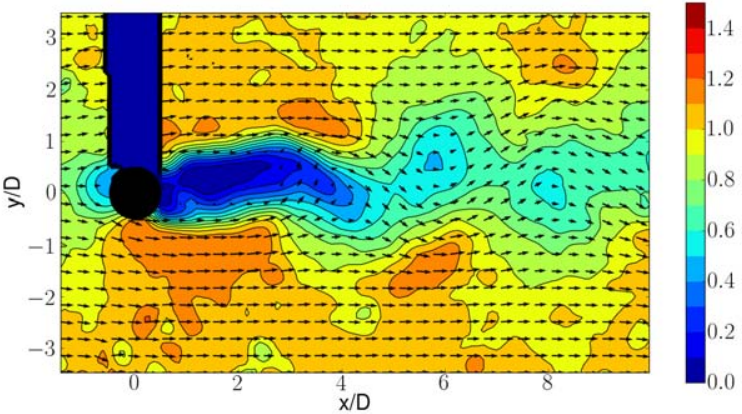

(b) Flow under DBD actuation.

Figure 3. Instantaneous PIV velocity fields. The region of interest contains $140 \times 86$ vectors, scaled to represent the flow more clearly. Contour levels corresponds to the velocity modulus.

Quantitative measurements were performed using 2D Particle Image Velocimetry (PIV) on a vertical plane placed at mid-span of the cylinder. Image acquisition and PIV calculation were done using a LaVision system, composed of an ImagerPro $1600 \times 1200 \mathrm{CCD}$ camera with a 12-bit dynamic range capable of recording double-frame pairs of images at $\sim 8 \mathrm{~Hz}$ and a two rod Nd:YAG $(15 \mathrm{~mJ})$ pulsed laser synchronized by a customized PC using LaVision DaVis 7.1 software. Laser sheet width was about $1 \mathrm{~mm}$ in the test section. The whole $300 \mathrm{~mm} \times 200 \mathrm{~mm}$ imaging region (about $12 \times 8 \mathrm{D}$ ) gives a spatial resolution of $0.06 \mathrm{D}$ as showed in Figure 3. All image acquisitions where done using the double-frame mode with a time lapse between the two frames $(\mathrm{d} t)$ set to $7 \mathrm{~ms}$.

Given the flow natural frequency, its dynamic behavior is well resolved by the PIV measures for the selected 
acquisition frequency.

\section{Problem definition.}

The geometry has been non-dimensionalized with the cylinder diameter $D$. The computational geometry is defined by a rectangle placed with dimensions $L=20$ and $H=49$. The center of the cylinder is placed at the middle of the box and 8 units ahead of the inflow boundary, see figure 4 .

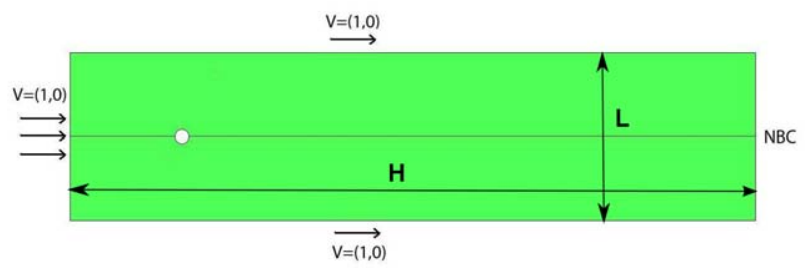

Figure 4. Computational setup: dimensions and boundary conditions.

The plasma actuation takes place in a thin layer compared to cylinder diameter. This enables to subdivide the problem into two main regions. An inner region where the forcing occurs and an outer region (where we will perform the numerical simulations) in which electric forces can be disregarded. A kinematic compatibility condition can be imposed at the boundary separating both regions. We assume in our case that normal velocity is null and that no discontinuity in tangential velocities exists. We further assume that the law that quantifies the tangential velocity distribution is governed from the parameters that determine the discharge in the inner region. In this work no modeling of the inner region based on plasma physics is proposed. The law is determined in our study directly from experiments. The boundary condition is approximated by the simplified log-normal function: ${ }^{17}$

$$
\mathbf{v}=A e^{f(x)+1-e^{f(x)} \tau}
$$

and

$$
f(x)=-\frac{2 x-x_{0}}{W}
$$

where $x$ varies from 0 to $D / 2, \tau$ is a tangential unit vector defined as clockwise at the top part of the cylinder and anticlockwise for the bottom part. The boundary velocity $\mathbf{v}(x)$ depends on three parameters: $A, W$ and $x_{0} . W$ is related to the pulse width, $A$ is related to the pulse amplitude and $x_{0}$ is related to the position of the maximum velocity, see figure 5 . In the present study $W=0.1$ and $x_{0}=0.35$ were fixed according to experimental measurements ${ }^{17}$ and the influence of the pulse amplitude $A$ was explored as a control parameter.

Thus for the outer problem the boundary can be divided in three parts: $\Gamma_{D}$ composed by the left(inflow), upper and lower boundaries where velocity $V=(1,0)$ has been prescribed; $\Gamma_{N}$ composed by the right(outflow) boundary where natural boundary conditions(NBC) have been used, (see equation 6) and for the cylinder boundary $\Gamma_{C}$ we prescribe a slip velocity imposed by the plasma actuator (in correspondence with the actuated thin layer), see equations (1-2).

\section{A. Base flow calculation.}

The modifications introduced by the plasma actuator are experimentally measured by the mean flow field variations. For this goal, 400 snapshots have been obtained which contains al least 30 shedding periods. We observe, in Figure 6 that the near wake region behind the cylinder enlarges under increasing voltage. The streamlines get close further downstream when it is applied the higher EHD actuation, resulting that the mean flow resembles more to the stationary solution, characterized by a long recirculation zone as it in Figure 1 scheme. 


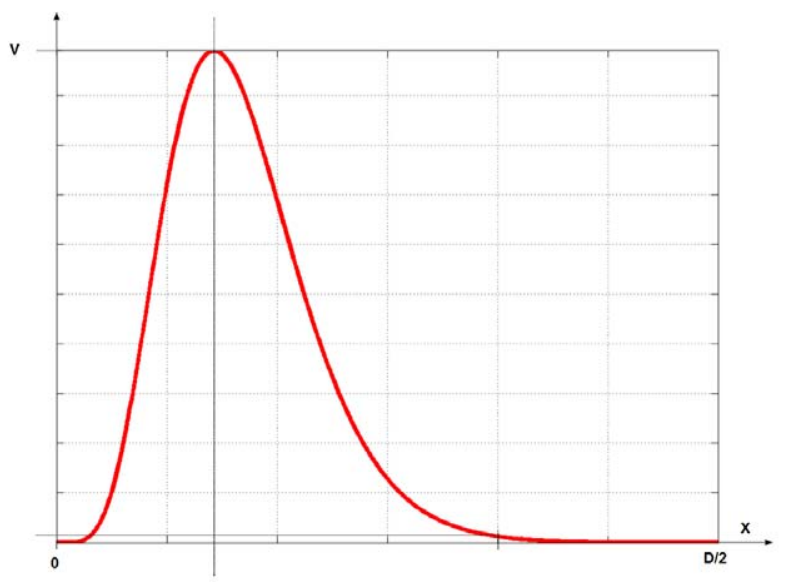

Figure 5. Representation of the boundary condition used to approximate the velocity profiles observed close to the wall when air is actuated. See equations (1-2)

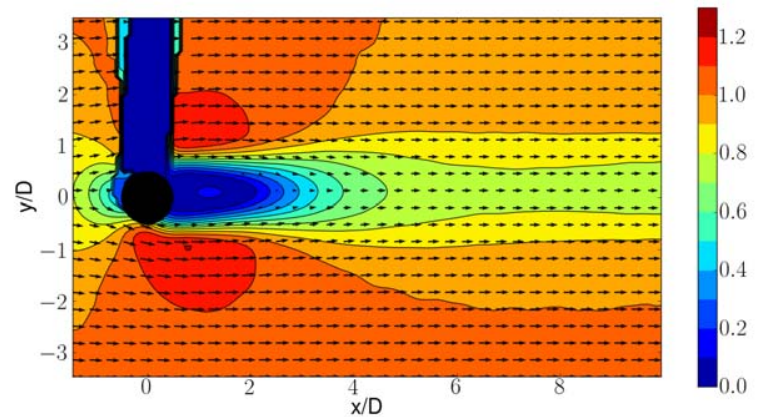

(a) Flow without forcing

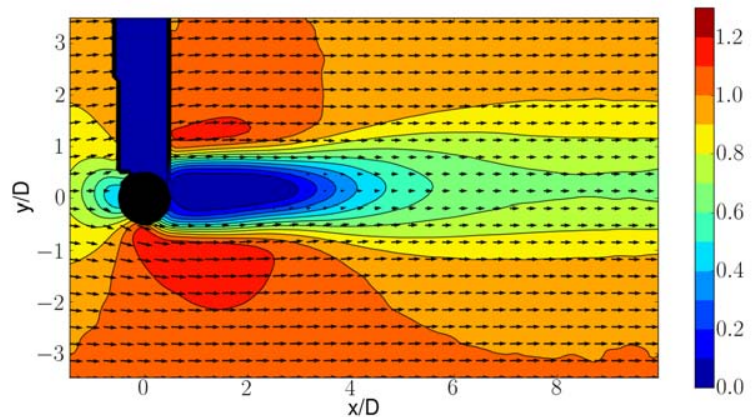

(c) Flow under DBD actuation. DC=16

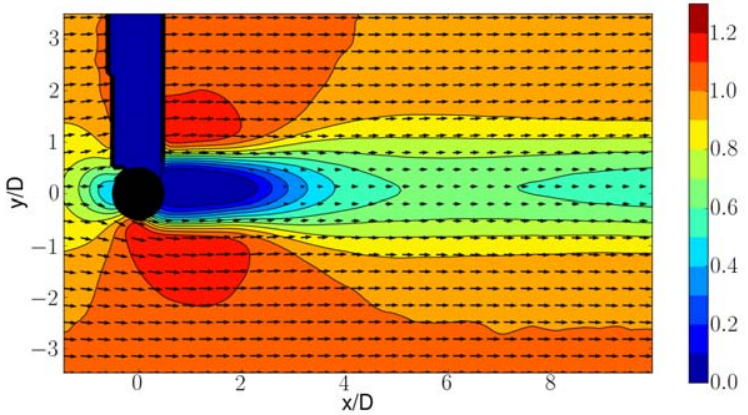

(b) Flow under DBD actuation. $\mathrm{DC}=10$

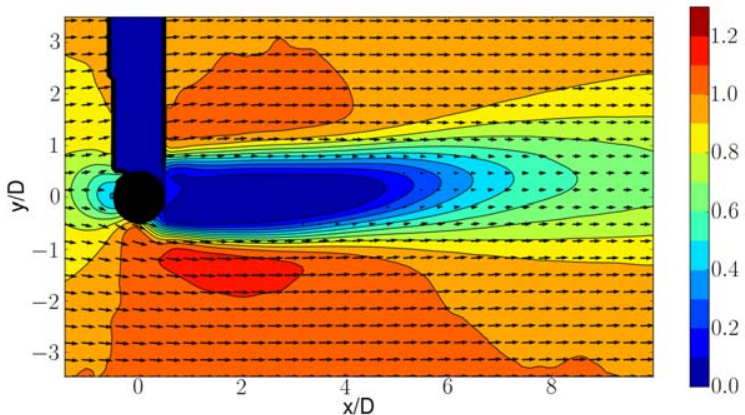

(d) Flow under DBD actuation. DC=22

Figure 6. Mean flow velocity vector field and velocity modulus in contours. 
First we calculate the mean flow with a time average over the total number of experimental snapshots obtained. Second, we determine the position $\left(x_{\max }, y_{\max }\right)$ where the root mean square of the velocity field is maximum. Third, we plot the root mean square of the velocity field for $y=y_{\max }$ along the horizontal axis. This third step is repeated for different values of the duty cycle (DC) parameter when DBD stationary forcing is used. We observe in Figure 7 that increments on DC leads to decrements in the mode amplitude and accordingly its position displaces downstream, proving the flow stabilization. From a certain threshold, the behavior is quite different as shows the curve corresponding to $\mathrm{DC}=28$.

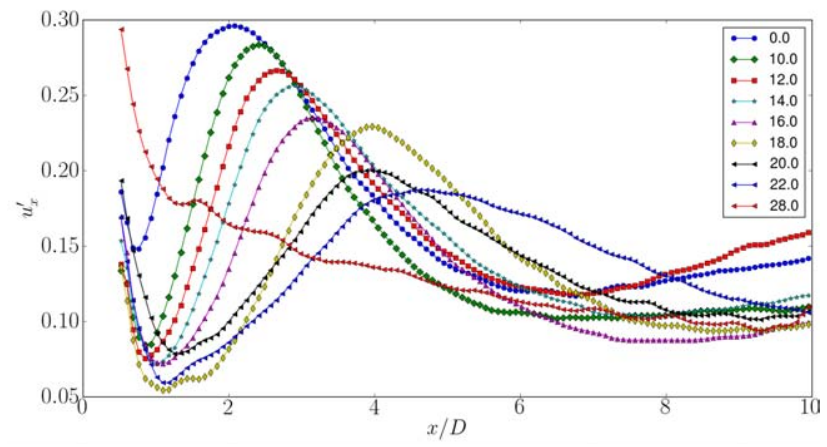

Figure 7. Amplitude of the velocity oscillations $u_{x}^{\prime}$ at $y=y_{\max }$ along the horizontal axis, for different values of the DBD forcing parameter (DC).

From the evolution of the oscillating amplitude, we can determine a critical value for the forcing parameter $D C$. From Figure 8(a), we confirm the hypothesis for the von Karman modes evolution when they are forced toward stabilization. The ratio $A_{\max } / X_{\max }$ decreases almost linearly from $D C=10$ up to $D C=22$ and we can estimate a critical value from the linear approximation $\left(D C_{C} \simeq 27.5\right)$. With this value we construct the scaling law for $\left(D C_{C}-D C\right)$ in Figure 8(b).

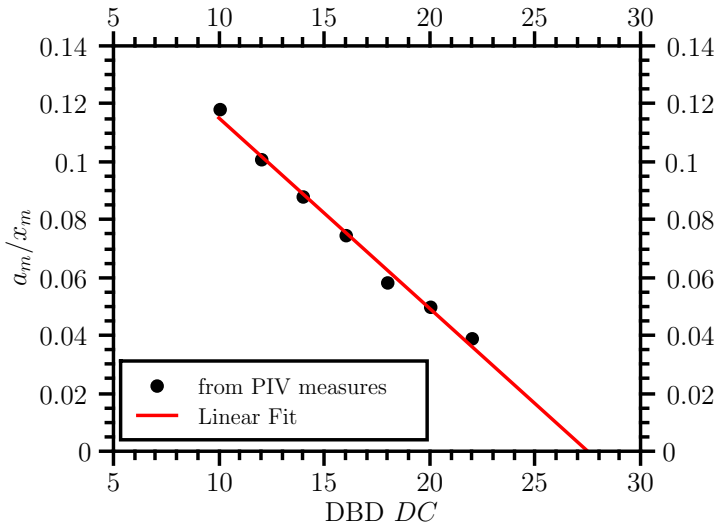

(a) Determination of critical value for DC

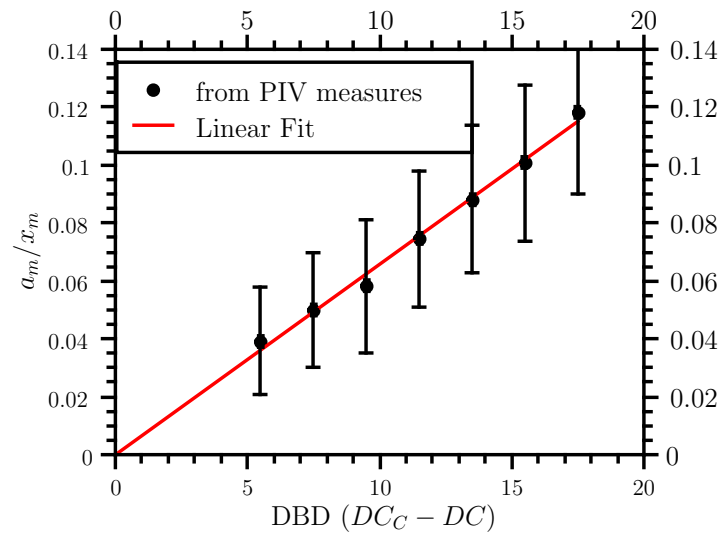

(b) Scaling law from $a_{m} / x_{m}$ evolution.

Figure 8. Scaling law for DC parameter.

We remark that further increases of DC parameter produces the flow to behave qualitatively different as we expected from our hypothesis. Figure 9 shows how Von Karman mode has been suppressed and fluctuations remain in the cylinder wall neighborhood. Indeed, the momentum added from the cylinder wall induces higher velocities and the velocity profiles downstream prevent self-sustained oscillations. The instability is confined to a region very close to the wall, Figure 9(a) shows an instantaneous velocity field for $\mathrm{DC}=28$ and Figure $9(\mathrm{~b})$ the mean flow for the corresponding forcing. 


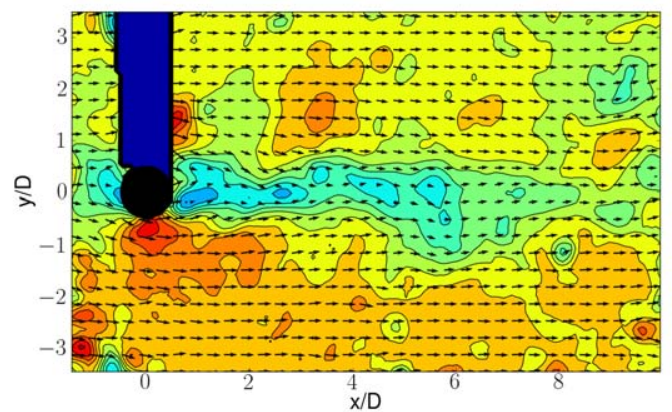

(a) Instantaneous

velocity Contours represents velocity modulus.

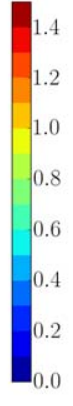

$\mathrm{DC}=28$. (b) Mean flow under DBD actuation $\mathrm{DC}=28$. tours represents velocity modulus.

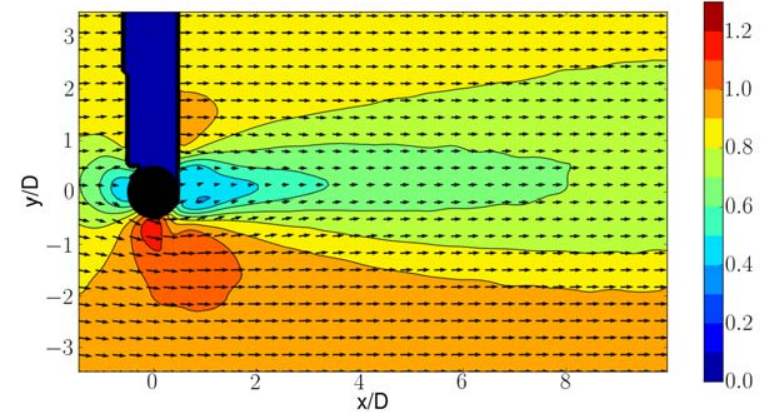

Con-

Figure 9. Beyond the bifurcation a qualitatively different dynamics takes place in the flow.

\section{Numerical study.}

The equations governing incompressible flows are written in primitive-variables formulation as follows

$$
\begin{aligned}
\frac{D \mathbf{u}}{D t} & =-\nabla p+\frac{1}{R e} \nabla^{2} \mathbf{u} \quad \text { in } \Omega, \\
\nabla \cdot \mathbf{u} & =0 \quad \text { in } \quad \Omega, \\
\mathbf{u} & =\mathbf{U} \quad \text { on } \quad \Gamma_{D}, \\
-p \mathbf{n}+\frac{1}{R e} \nabla \mathbf{u} \cdot \mathbf{n} & =0 \quad \text { on } \quad \Gamma_{N} .
\end{aligned}
$$

Here $\Omega$ is the computational domain, $\Gamma_{D}$ is the part of its boundary where Dirichlet boundary conditions represented by $\mathbf{U}$ are imposed, $\Gamma_{N}$ is the part of the boundary where the "natural" boundary conditions (6) are imposed, $n$ is the vector normal to the boundary $\Gamma_{N}$, the material derivative operator is defined as usual, $\frac{D}{D t}=\frac{\partial}{\partial t}+u_{j} \frac{\partial}{\partial x_{j}}$, and repeated indices imply summation.

\section{A. The steady laminar basic flows}

The two-dimensional equations of motion are solved in the laminar regime at appropriate $R e$ regions, in order to compute steady basic flows $(\overline{\mathbf{u}}, \bar{p})$ whose stability will subsequently be investigated. The equations read

$$
\begin{aligned}
& \frac{D \overline{\mathbf{u}}}{D t}=-\nabla \bar{p}+\frac{1}{R e} \nabla^{2} \overline{\mathbf{u}} \quad \text { in } \quad \Omega, \\
& \nabla \cdot \overline{\mathbf{u}}=0 \quad \text { in } \quad \Omega \text {, } \\
& \overline{\mathbf{u}}=\mathbf{f} \text { on } \Gamma_{D}, \\
& -\bar{p} \mathbf{n}+\frac{1}{R e} \nabla \overline{\mathbf{u}} \cdot \mathbf{n}=0 \quad \text { on } \quad \Gamma_{N} .
\end{aligned}
$$

In the problem solved in what follows, the basic flow velocity vector is $\left(\bar{u}_{1}, \bar{u}_{2}, 0\right)^{\mathrm{T}}$, i.e. its component along the spatial direction $x_{3}$ is taken to be zero $\bar{u}_{3}=0$, and all components are taken to be independent of this spatial direction, $\frac{\partial \bar{u}_{i}}{\partial x_{3}}=0$. The consequence is that the linearized equations of motion defining the BiGlobal stability problem to be solved may be expressed by real operators, as will be discussed shortly. In this case the basic flow is obtained by time-integration of the system (7-8) by a Semi-Lagrangian second order finite element solver ADFC, ${ }^{18}$ starting from rest and being driven by the boundary conditions. 


\section{B. The linear systems}

The basic flow is perturbed by small-amplitude velocity $\tilde{u}_{i}$ and kinematic pressure $\tilde{p}$ perturbations, as follows

$$
\mathbf{u}=\overline{\mathbf{u}}+\varepsilon \tilde{\mathbf{u}}+\text { c.c. } \quad p=\bar{p}+\varepsilon \tilde{p}+\text { c.c. }
$$

where $\varepsilon \ll 1$ and c.c. denotes conjugate of the complex quantities $(\tilde{\mathbf{u}}, \tilde{p})$. Substituting into equations (34 ), subtracting the basic flow equations (7-8), and linearizing, the equations for the perturbation quantities are obtained

$$
\begin{aligned}
\frac{\bar{D} \tilde{\mathbf{u}}}{D t}+\tilde{\mathbf{u}} \nabla \overline{\mathbf{u}} & =-\nabla \tilde{p}+\frac{1}{R e} \nabla^{2} \tilde{\mathbf{u}}, \\
\nabla \cdot \tilde{\mathbf{u}} & =0
\end{aligned}
$$

with $\frac{\bar{D}}{D t}=\frac{\partial}{\partial t}+\bar{u}_{j} \frac{\partial}{\partial x_{j}}$. The boundary conditions used for this system are

$$
\begin{array}{rlrlrl}
\tilde{\mathbf{u}} & =0 & & \text { on } & & \Gamma_{D} \cup \Gamma_{C} \\
-\tilde{p} \mathbf{n}+\frac{1}{R e} \frac{\partial \tilde{\mathbf{u}}}{\partial n} & =0 & \text { on } & & \Gamma_{N} .
\end{array}
$$

\section{Eigenvalue Problem (EVP) formulation and solution methodology}

The separability of temporal and spatial derivatives in (12-13) permits introduction of an explicit harmonic temporal dependence of the disturbance quantities into these equations, according to the Ansatz

$$
\begin{aligned}
& \tilde{\mathbf{u}}=\hat{\mathbf{u}}(x, y) e^{i \beta z} e^{\omega t}, \\
& \tilde{p}=\hat{p}(x, y) e^{i \beta z} e^{\omega t},
\end{aligned}
$$

where a temporal formulation has been adopted, considering $\beta$ a real wavenumber parameter, while $\omega$ is the complex eigenvalue sought. Substitution into (12-13) results in

$$
\begin{aligned}
\left\{\bar{u}_{j} \frac{\partial}{\partial x_{j}}+\frac{\partial \bar{u}_{1}}{\partial x}+i \beta \bar{u}_{3}-\frac{1}{R e}\left(\frac{\partial^{2}}{\partial x_{j}^{2}}-\beta^{2}\right)\right\} \hat{u}_{1}+\hat{u}_{2} \frac{\partial \bar{u}_{1}}{\partial y}+\frac{\partial \hat{p}}{\partial x} & =-\omega \hat{u}_{1}, \\
\left\{\bar{u}_{j} \frac{\partial}{\partial x_{j}}+\frac{\partial \bar{u}_{2}}{\partial y}+i \beta \bar{u}_{3}-\frac{1}{R e}\left(\frac{\partial^{2}}{\partial x_{j}^{2}}-\beta^{2}\right)\right\} \hat{u}_{2}+\hat{u}_{1} \frac{\partial \bar{u}_{2}}{\partial x}+\frac{\partial \hat{p}}{\partial y}= & -\omega \hat{u}_{2}, \\
\left\{\bar{u}_{j} \frac{\partial}{\partial x_{j}}+i \beta \bar{u}_{3}-\frac{1}{R e}\left(\frac{\partial^{2}}{\partial x_{j}^{2}}-\beta^{2}\right)\right\} \hat{u}_{3}+\hat{u}_{1} \frac{\partial \bar{u}_{3}}{\partial x}+\hat{u}_{2} \frac{\partial \bar{u}_{3}}{\partial y}+i \beta \hat{p} & =-\omega \hat{u}_{3}, \\
\frac{\partial \hat{u}_{1}}{\partial x}+\frac{\partial \hat{u}_{2}}{\partial y}+i \beta \hat{u}_{3} & =0 .
\end{aligned}
$$

In the case of a basic flow velocity vector with components $\left(\bar{u}_{1}, \bar{u}_{2}, 0\right)$ only on the plane normal to the wavenumber vector, it is possible to deduce a real eigenvalue problem, by the redefinition of the out-of-plane velocity component ${ }^{19}$

$$
\hat{u}_{3} \equiv i \hat{u}_{3} .
$$

This converts the system (18-21) into one with real coefficients. Defining

$$
\alpha_{i i}=\left\{\bar{u}_{j} \frac{\partial}{\partial x_{j}}+\frac{\partial \bar{u}_{i}}{\partial x_{i}}-\frac{1}{R e}\left(\frac{\partial^{2}}{\partial x_{j}^{2}}-\beta^{2}\right)\right\}, \quad j=1,2 .
$$

(no Einstein summation implied for index $i$ ), the left-hand side of the system can be represented by the real non-symmetric operator $A$ as 


$$
A=\left(\begin{array}{cccc}
\alpha_{11} & \frac{\partial \bar{u}_{1}}{\partial y} & 0 & \frac{\partial}{\partial x} \\
\frac{\partial \bar{u}_{2}}{\partial x} & \alpha_{22} & 0 & \frac{\partial}{\partial y} \\
0 & 0 & \alpha_{33} & -\beta \\
\frac{\partial}{\partial x} & \frac{\partial}{\partial y} & \beta & 0
\end{array}\right)
$$

After the variational formulation, details of which are presented in appendix, the real operator $A$, which is represented by a $(3 N+N L)^{2}$ matrix, becomes

$$
A=\left(\begin{array}{cccc}
F_{i j}+C_{i j}^{11} & C_{i j}^{12} & 0 & -\lambda_{i j}^{x} \\
C_{i j}^{21} & F_{i j}+C_{i j}^{22} & 0 & -\lambda_{i j}^{y} \\
0 & 0 & F_{i j} & -\beta D_{i j} \\
\lambda_{j i}^{x} & \lambda_{j i}^{y} & \beta D_{j i} & 0
\end{array}\right) \quad i, j=1, \cdots 4 .
$$

where $F_{i j} \equiv \gamma_{i j}+\left(R_{i j}+\beta^{2} M_{i j}\right)$. The real symmetric operator $B$ is also introduced by

$$
B=\left(\begin{array}{cccc}
M_{i j} & 0 & 0 & 0 \\
0 & M_{i j} & 0 & 0 \\
0 & 0 & M_{i j} & 0 \\
0 & 0 & 0 & 0
\end{array}\right), i, j=1, \cdots, N
$$

where $M$ represents the mass matrix; the elements of all matrices introduced in (24) and (25) are presented in the appendix. The system (18-21) is thus transformed into the real generalized eigenvalue problem for the determination of $\omega$,

$$
A\left(\begin{array}{c}
\hat{u}_{1} \\
\hat{u}_{2} \\
\hat{u}_{3} \\
\hat{p}
\end{array}\right)=-\omega B\left(\begin{array}{c}
\hat{u}_{1} \\
\hat{u}_{2} \\
\hat{u}_{3} \\
\hat{p}
\end{array}\right)
$$

The real generalized eigenvalue problem (26) has either real or complex solutions, corresponding to stationary $\left(\omega_{i}=0\right)$ or traveling $\left(\omega_{i} \neq 0\right)$ modes. Since the operators $A$ and $B$ are real in this class of problems, the generalized eigenvalue problem (26) has either real or complex conjugate pairs of solutions. On the other hand, real arithmetic suffices for the calculation and storage of (and subsequent operations with) the nonzero elements of the matrices $A$ and $B$.

From a linear stability analysis point of view, the most important eigenvalues are those closest to the axis $\omega_{r}=0$ and here an iterative method has been used for their determination. Specifically, the well-established in BiGlobal linear instability problems ${ }^{19}$ Arnoldi algorithm has been used, see ${ }^{20}$ for details.

All computations have been performed serially on a $3.0 \mathrm{GHz}$ Intel P-IV PC. A typical leading dimension of matrix $A$ used in the present analyses is $\operatorname{DIM}(A) \equiv 3 N+N L=O\left(7 \times 10^{4}\right)$, while only the nonzero elements of this matrix, $O\left(9 \times 10^{6}\right)$, and those of its LU decomposition are stored.

\section{Results.}

\section{A. Base flow results.}

The first task is to compare the computational results obtained for a forcing parameter $A$ with the experimental results obtained when the $D C$ parameter was varied. In figure 10, the horizontal components of the computationally calculated flow are shown for $A=0.5$ and $A=1$. If we look at the cylinder perimeter the maximum actuation area can be appreciated.

In figure 11 different wake cuts are shown for different couples of control parameters $A=1,1.5,2$ and $D C=6,12,30$. For each cut the computational and the average experimental velocity have been compared at $R e=235$. As we can appreciated from the figures the agreement between the experimental measurements 

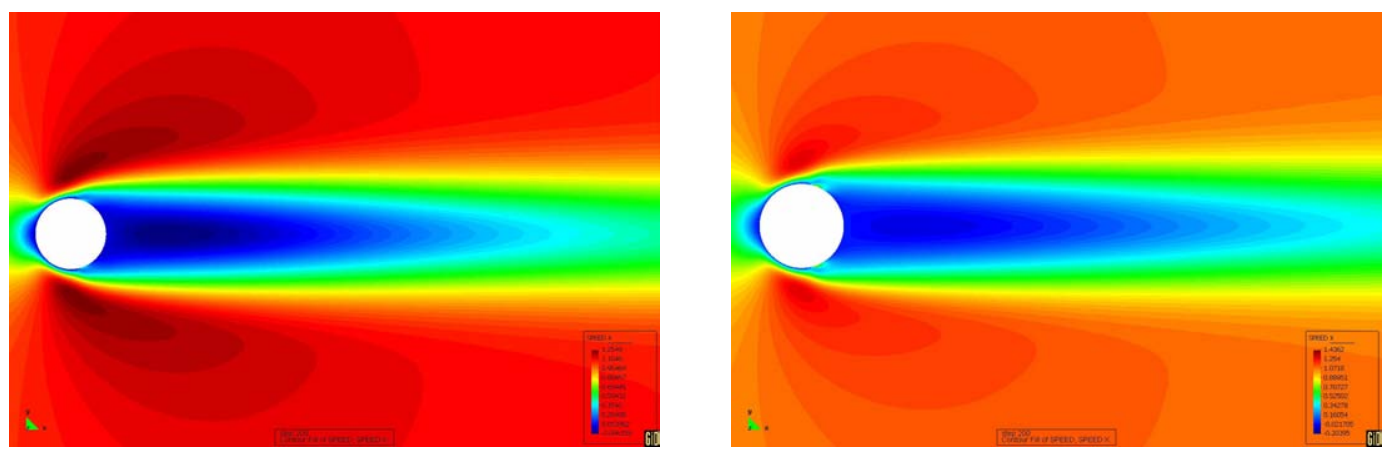

Figure 10. Horizontal component of the stationary baseflow velocity field $u_{1}$ for $\mathbf{A}=0.5($ right $)$ and $\mathbf{A}=1($ left $)$ computed by ADFC

and the computed values is quite good, specially taking into account the simplicity of the boundary control model that mimics the presence of the plasma actuator.

In figures 12 and 13 the root mean square of the experimental and the computational have been calculated for different values of the respective control parameters $A$ and $D C$ when the Reynolds number has been kept fixed at $R e=235$. In figure 12 , we can appreciate that when the parameter $A$ is increased the root mean square decreases and the area of maximum variation moves away from the cylinder. It is also remarkable to observe how beyond a certain critical value of the control parameter $A$ (two highest values), the root mean square of the flow falls to zero.

The efficiency of the control parameter can be also appreciated when the root mean square is calculated from the experimental values, see figure 13. The maximum oscillation moves towards higher $x$ values and its value decreases when the $D C$ is increased. This tendency is maintained until we reach the highest value of the control parameter when the structure of the flow changes drastically. We can assume that experimentally a zero root mean square flow is not possible due to the intrinsic instability of the perturbed inflow. Therefore, the critical change in the experimental configuration must be detected when the root mean square curve has an abrupt change.

Using the comparison results obtained before, the corresponding values of $A$ and $D C$ are plotted in figure 14. This picture shows, one of the most important results of this work where the two control parameters, computational and experimental, are finally related.

Once the control parameters have been related when can plot the root mean square evolution for both approaches. In figure 15, the evolution of the root mean square with the control parameter is monitored computationally and experimentally. As we can appreciated the linear fit of both approaches give a critical parameter which is not far from the other.

\section{B. Vortex suppression and Hopf bifurcation.}

As a validation case for the computational stability code, the critical Reynolds number when no control is acting has been tested. As it was obtained in the seminal paper by Barkley and Henderson, ${ }^{21}$ a critical Reynolds number $R e=47$ has been obtained, in excellent agreement with previous studies.

For other flows where the control parameter is not zero, the value of $A$ has been increased from $A=0.1$ until $A=2$. The stability analysis is able to calculate the damping rate and the frequency of a large number of flow perturbation modes. In figure 16 the frequency of the least stable mode and the damping rate of that mode have been represented. Two conclusions can be obtained from these figures, first the frequency of the least stable mode increases when the control parameter is increased. Second, the damping rate shows a linear dependance on the Reynolds number when the control parameter is kept constant.

The main result of our investigation is illustrated in figure 17, where the neutral stability curve in the $(R e, A)$ plane has been plotted and the stability regions are distinguishable. The main conclusion inferred from figure 17 is that the plasma control mechanism delays the onset vortex shedding. The actuator effect is significant, and small increments of the control parameter $A$ lead to large changes in the critical Reynolds number, with a very sharp increase for $A>1.5$. 

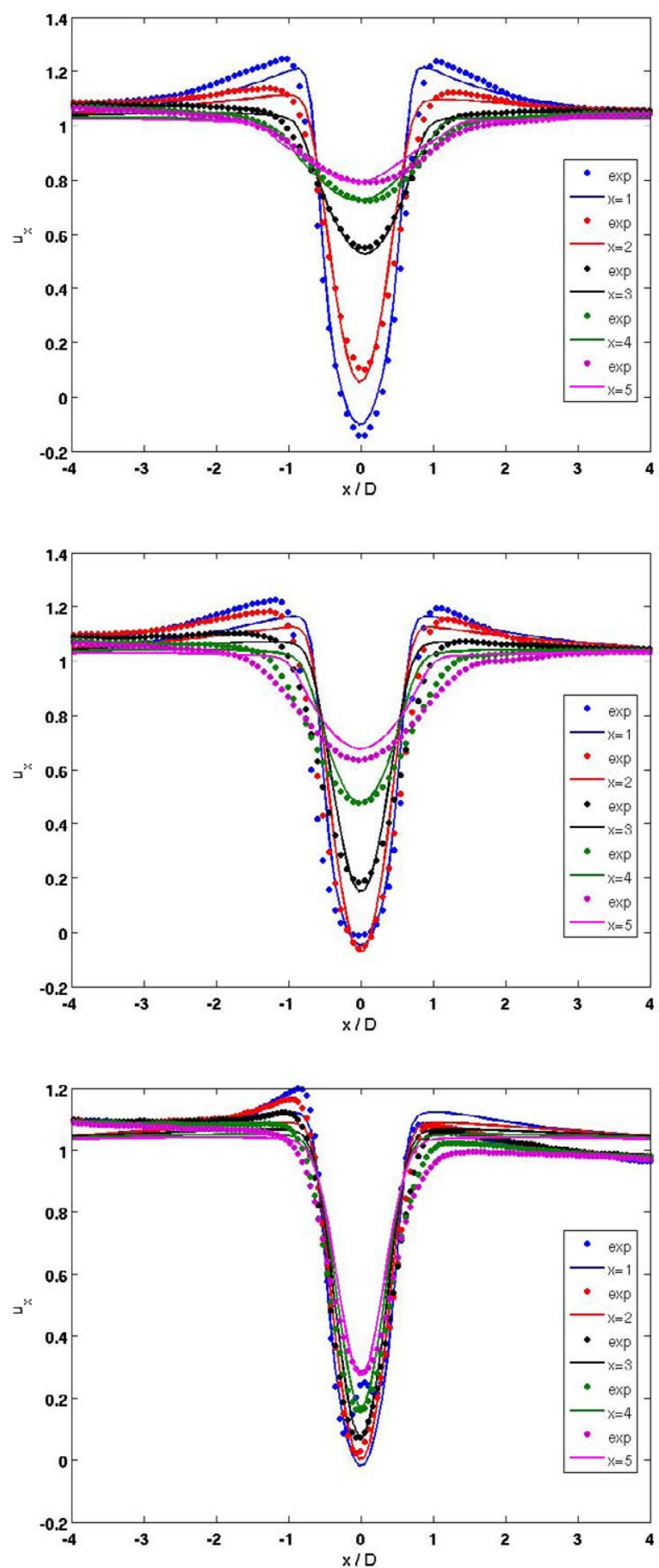

Figure 11. Comparison of the horizontal average velocity profiles at $A=1($ top), $A=1.5$ (middle) and $\mathrm{A}=\mathbf{2}$ (bottom) 


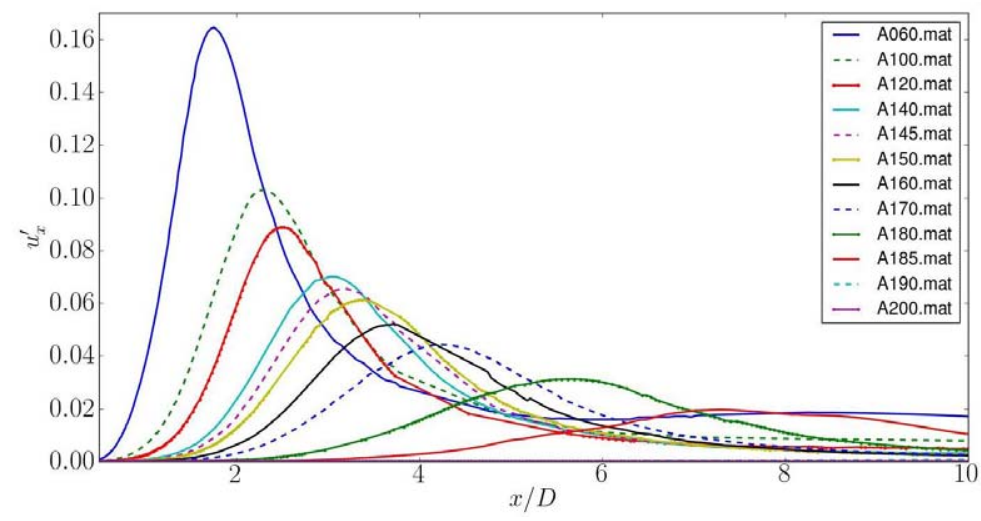

Figure 12. Root mean square of the velocity profiles with numerical results. $A$ varies from 0.6 to 2.

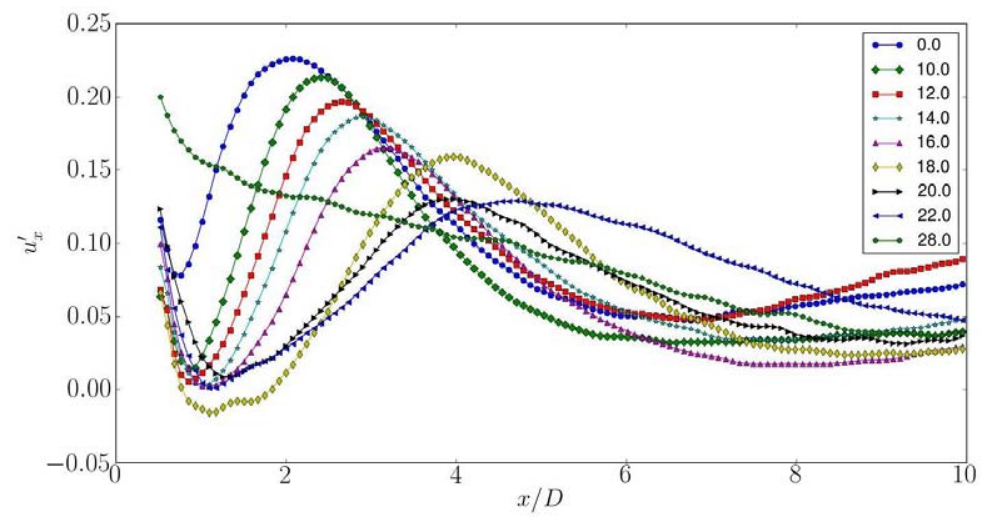

Figure 13. Root mean square of the velocity profiles with experimental results. DC varies from 0 to 28 .

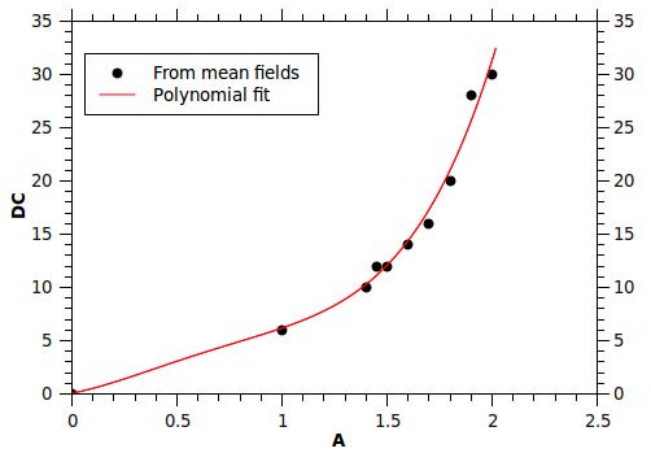

Figure 14. Correlation between the experimental and computational control parameters A and DC. 


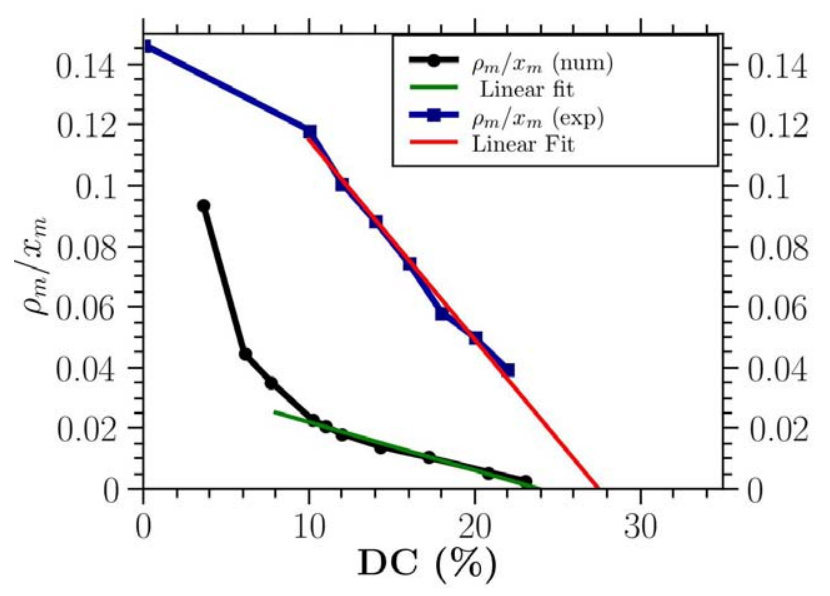

Figure 15. Dependance of the root mean square with the forcing parameter.
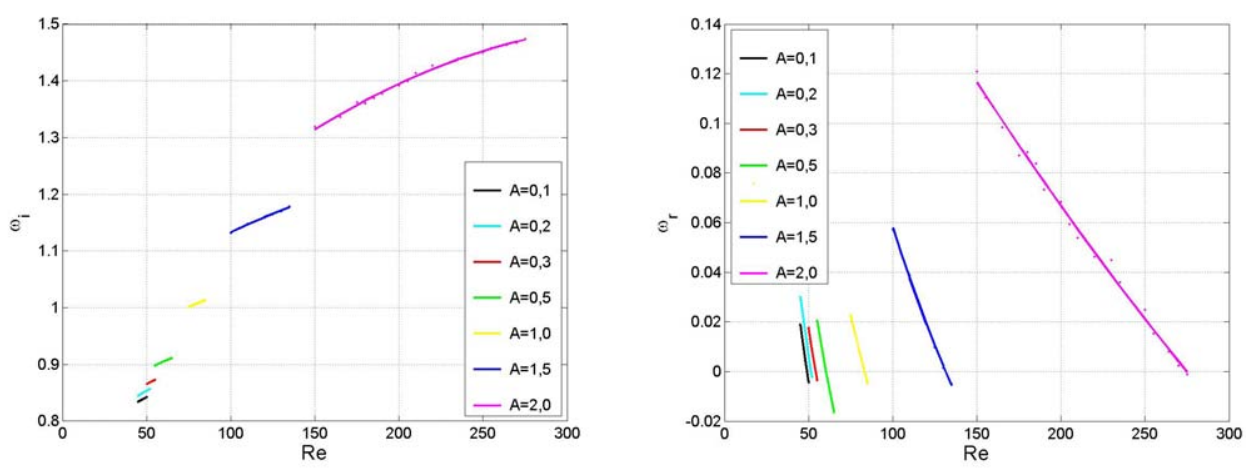

Figure 16. Frequency(left) and damping rate(right) of the least stable mode vs. Reynolds number

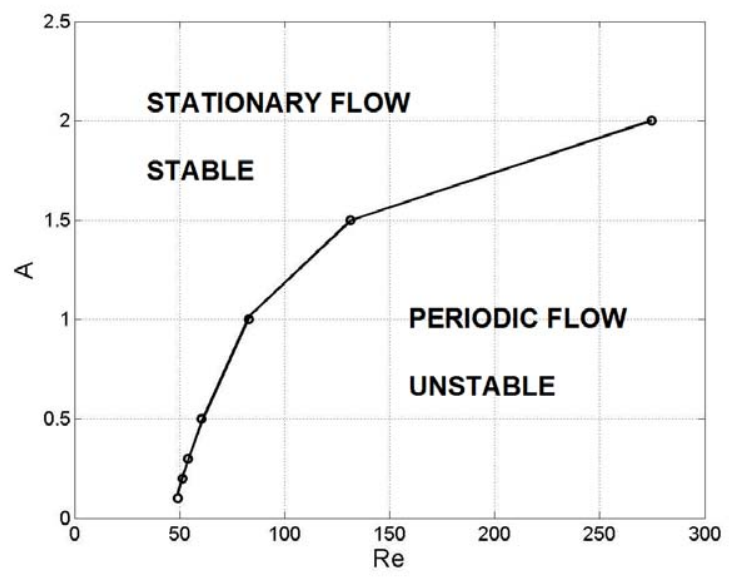

Figure 17. Control parameter $A$ vs. critical Reynolds number.

\section{3 of 18}



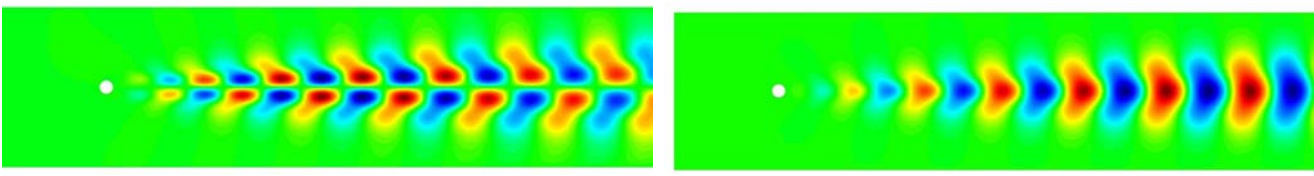

i.
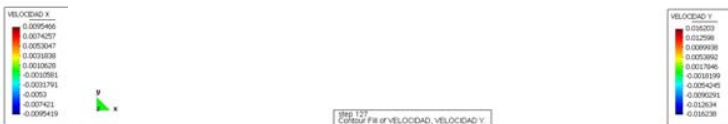

Figure 18. Horizontal(left) and vertical(right) components of the velocity perturbation $\widehat{u}_{1}$ and $\widehat{u}_{2}$ for $\mathbf{A}=0.5$
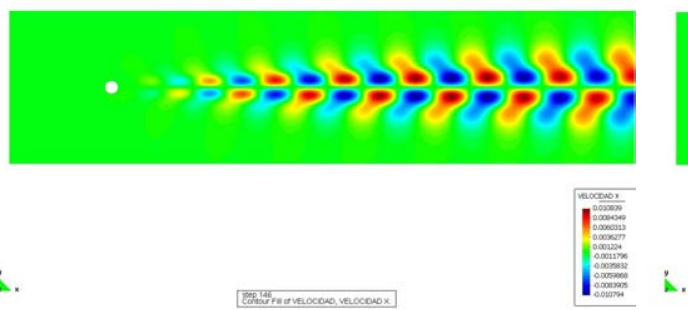
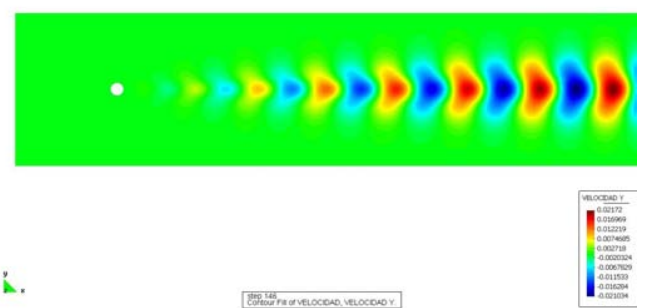

Figure 19. Horizontal(left) and vertical(right) components of the velocity perturbation $\widehat{u}_{1}$ and $\widehat{u}_{2}$ for $\mathbf{A}=1$

\section{Physical interpretation.}

Following the steps of ${ }^{22}$ and $^{14}$ we have checked if the interpretation of the vortex shedding as a critical accumulation of surface vorticity could be supported with our local control mechanism. First, we should point out that the control mechanism suggested by Legendre et al. is a slip boundary condition on the surface of the cylinder, this mechanism acts globally on the the whole perimeter, which is quite different to our local actuator that acts locally in a small part of the cylinder. In the vorticity curve, see figure 20, we are able to see that a local control modifies drastically the vorticity distribution and thus a modified criteria to interpret the vortex shedding should be used. The actuator introduces a local singularity in the vortex distribution that perturbs drastically the tendency presented in the vorticity distribution in control absence. Different to what happens in the slip control mechanism, for a given $R e$ number increasing $A$ leads to a increase of the vorticity intensity in the boundary layer, see 20. Similarly to the slip control, the wake due to the decrease of the separation angle is narrowed, see figure 21 . In the case $R e=100$, the separation angle varies from 25.3 for $A=1$ to 16.1 for $A=1.5$.

The vorticity generated in the cylinder surface is generated in the cylinder by two mechanisms: first, the classical vorticity distribution when no control mechanism is added to the system plus a second vorticity distribution due to the plasma actuation.

This accumulated vorticity is transported from the perimeter of the cylinder to the wake. As Legendre points out in ${ }^{14}$ there are strong indications that the formation of a standing eddy and the occurrence of wake instability arises when the vorticity accumulated $\Omega_{a c c}$ around the body exceeds some critical value. This vorticity accumulation must be understood as the vorticity contained in the stationary recirculating wake flow. This vorticity accumulated $\Omega_{a c c}$ is the difference between the vorticity generated at the cylinder surface $\Omega_{\text {prod }}$ and the vorticity evacuated downstream $\Omega_{\text {evac }}$. Let us call $\omega$ the vorticity per unit volume, then:

$$
\Omega=\int_{S} \omega d s
$$

where $S$ a stationary recirculating flow area.

The $2 \mathrm{D}$ vorticity transport equation $D \omega / D t=\nu \nabla^{2} \omega$ must be written in integral form as: 


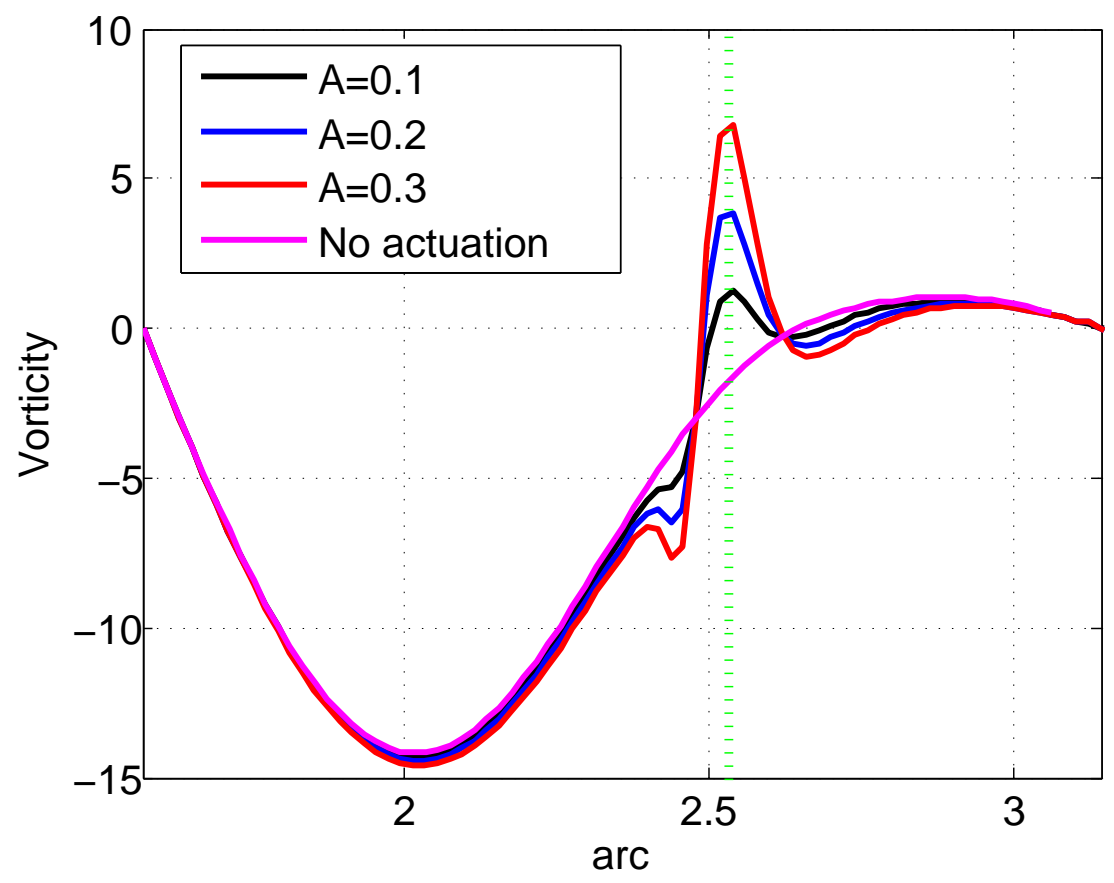

Figure 20. Vorticity intensity at $R e=50$ for the upper half of the cylinder, the arc parameter $s \in[\pi / 2, \pi]$. The dot green vertical line references the maximum control actuation.

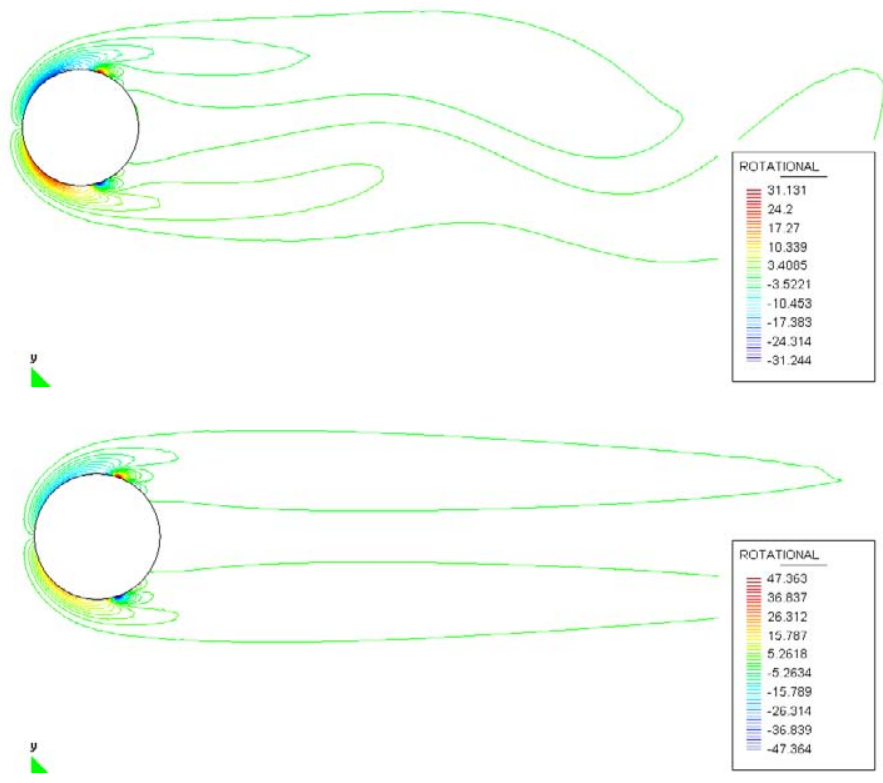

Figure 21. Vorticity contour lines at $R e=100$ at $A=0.5$ (top) and $A=1$ (bottom). 


$$
\int_{S} \frac{\partial \omega}{\partial t} d s+\oint_{L} \omega(\mathbf{v} \cdot \mathbf{n}) d l=\int_{S} \nu \nabla^{2} \omega d s
$$

where $L$ is the closed material curve that limits the recirculation area $S$. Using also the Gauss theorem for the viscous term, we have:

$$
\int_{S} \frac{\partial \omega}{\partial t} d s=\oint_{L}(\nu \nabla \omega \cdot \mathbf{n}-\omega \mathbf{v} \cdot \mathbf{n}) d l
$$

On the left hand side we have the accumulated vorticity in our recirculating flow area taking into account the viscous dissipation, on the other side we have the difference between the injected vorticity and the evacuated vorticity. This equation can be simplified as:

$$
\Omega_{a c c}=\Omega_{\text {prod }}-\Omega_{\text {evac }}
$$

According to Legendre when $\Omega_{a c c}$ exceeds a critical value the vortex shedding occurs. In the absence of control the accumulated vorticity can be related to the maximum value of the vorticity at the wall. The criterium that sustains that stability is related to the maximum accumulated vorticity can then be expressed in terms of a maximum value of vorticty at the wall. In our case, under forcing conditions the criterium based on the maximum vorticity at the cylinder surface $\omega_{\max }$ does not work so straight forward and it should be modified. With our plasma control mechanism the vorticity distribution is far from being smooth as in the slip case and the singularity introduced by the actuator breaks the natural vorticity transport mechanism. The vorticity region that feeds the recirculation area is basically coming from the vorticity accumulated in the body surface, when the plasma control is introduced a vorticity maximum appears in the cylinder surface. The presence of this local vorticity maximum in the cylinder surface prevents the natural transport mechanism from the production area to the wake area. The local maximum in the vorticity due to the actuator changes the sign of the vorticity gradient in a local area and consequently the natural transport mechanism. In this paper the maximum vorticity on the body surface criterium has been tested, see figure 22 , but the results obtained do not present such a concluding aspect as the ones presented by Legendre. The maximum dimensionless vorticity results higher when the control parameter increases. Thus applying the criterium of maximum vorticity at the wall and contrary to numerical simulation results and experiments the flow could not be stabilized when increasing actuation. Probably, and future works should point this direction, a more complex criterion inspired in the right hand side terms of the equation 29 should be defined. This means that with the plasma control used here, is not easy to define such criterion and the linear stability analysis is the most appropriate tool to predict the vortex shedding.

It is worth mentioning that another big advantage of the linear stability analysis performed here is that the transition from two-dimensional flow to three dimensional flow can be easily studied by changing the value of the $\beta=2 \pi / L_{z}$ parameter. In this work, in all flows close to the critical conditions, the $\beta$ parameter has been varied from $\beta=[0,10 D]$ and no three-dimensional instability has been found. This result means that, different to the work performed by Legendre ${ }^{14}$ where the two-dimensionality of the flow was taken as an assumption, in this case the possible three-dimensional transition is taken into account in the analysis and no evidence was found.

\section{Conclusions.}

In this work, we performed experimental and direct numerical simulations to study the influence of a plasma actuator on the dynamics of the two-dimensional wake behind a circular cylinder. The plasma actuation takes place in a thin layer compared to cylinder diameter. This enables to subdivide the problem in two regions. A thin inner region where the forcing occurs and an outer region where electric forces can be disregarded. A kinematic comptability condition has to be imposed at the boundary separating both regions. We assume in our case that normal velocity is null and that no discontinuity in tangential velocities exists. No modelling of the inner region is proposed and the mathematical law that quantifies the tangential velocity distribution is determined from experiments. The presence of the plasma control delays the vortex shedding in the wake, makes the recirculation zone longer, alters the vorticity distribution on the cylinder and increases the shedding frequency. The critical control parameters where the vortex shedding is obtained

has been studied as a function of the Reynolds number with a linear stability analysis of the Navier-Stokes equations. The derived generalized eigenvalue problem has been solved by an iterative Arnoldi method 


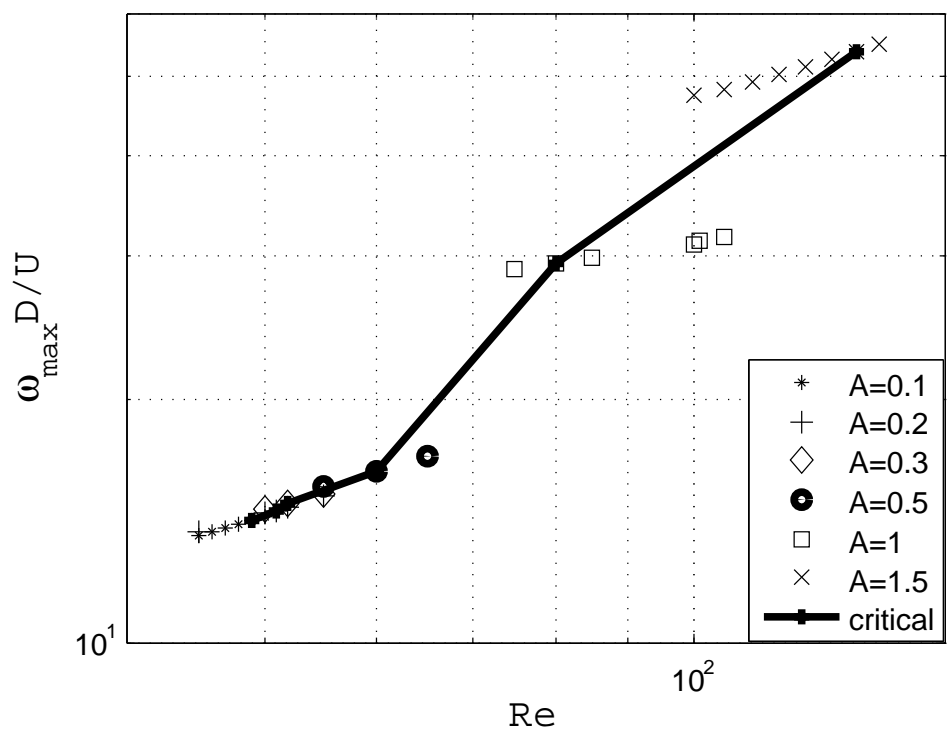

Figure 22. Maximum dimensionless vorticity $\omega D / U$ at the cylinder surface as a function of the Reynolds number $(\log -\log$ scale).

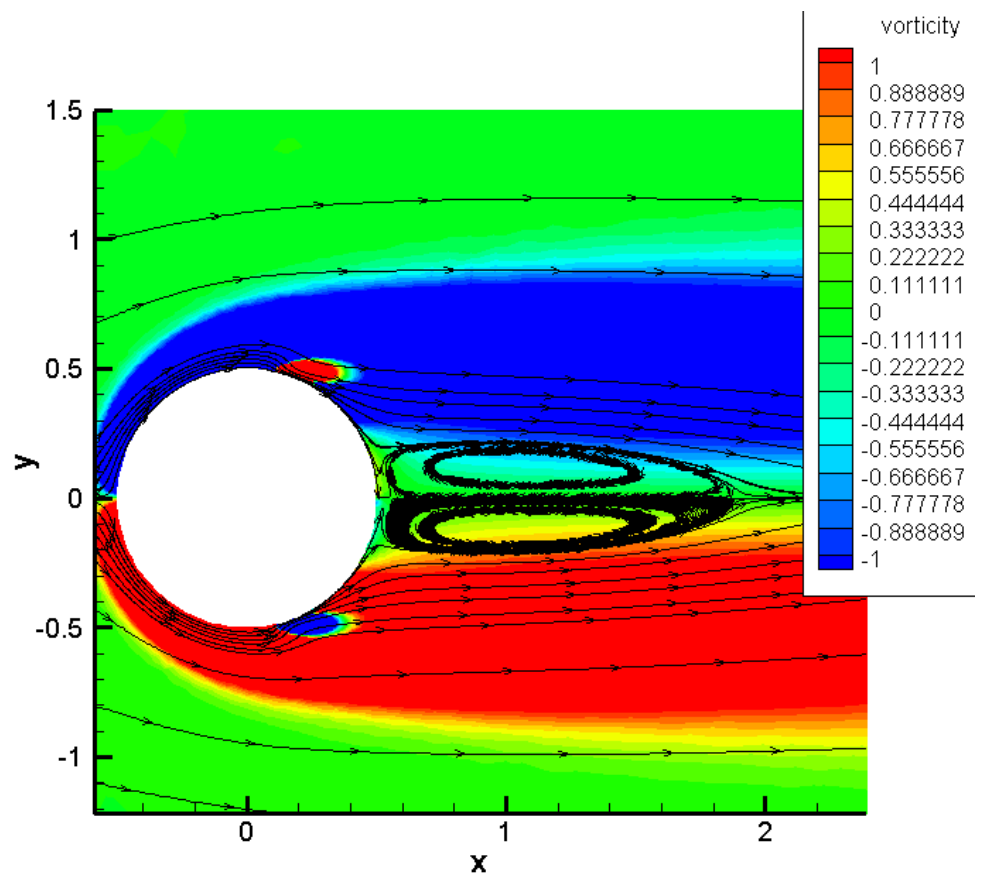

Figure 23. Vorticity and streamlines at $R e=100$ and $A=1.5$ (stable case). 
and the critical modes, damping rates and frequencies of the dominant modes of the spectrum have been obtained.

This three dimensional linear stability approach is also able to predict if the flow turns from 2D into a 3D flow and we finally concluded that in our Reynolds number range no unstable 3D transition has been found. Therefore the presence of the actuator keeps the flow two-dimensional for all cylinder lengths.

The neutral stability curve that separates the stable region where no vortex shedding is present from the other periodic region has been calculated and a good agreement has been obtained with experiments.

\section{Acknowledgments}

We would like to acknowledge the financial support made available by the Universidad Politécnica de Madrid through the Mobility Research Programm UPM-2010. Leo M. González wants to thank Eusebio Valero for the financial support to attend this Conference.

\section{References}

${ }^{1}$ Zielinska, B. and Wesfreid, J., "On the spatial structure of global modes in wake flow," Physics of Fluids, Vol. 7, 1995, pp. 1418.

${ }^{2}$ Wesfreid, J., Goujon-Durand, S., and Zielinska, B., "Global mode behavior of the streamwise velocity in wakes." Journal de Physique Paris II, Vol. 6, 1996., pp. 1343-1357.

${ }^{3}$ Moreau, E., "Airflow control by non thermal plasma actuators," J. Phys. D: Appl. Phys., Vol. 40, 2007, pp. 605-36.

${ }^{4}$ S. Masuda, M. W., "Ionic charging of very high resistivity spherical particle," J. Electrostatics, Vol. 6, 1979, pp. 57-67.

${ }^{5}$ Roth, J., Sherman, D., and Wilkinson, S., "Boundary layer flow control with a one atmosphere uniform glow discharge surface plasma," AIAA Meeting (Reno, USA, January 1998) paper \#98-0328, 1998.

${ }^{6}$ Thomas, F., Koslov, A., and Corke, T., "Plasma actuators for bluff body flow control," AIAA Meeting (San Francisco, USA, June 2006) paper \#2006-2845, 2006.

${ }^{7}$ Gonzalez, L. M., Artana, G., Gronskis, A., and D'Adamo, J., "A computational moving surface analogy of an electrodynamic control mechanism in bluff bodies," Global Flow Instability and Flow Control IV, Crete, Greece, September 2009, 2009.

${ }^{8}$ Gronskis, A., "Modelos numericos para el estudio de flujos externos controlados con actuadores EHD," PhD Thesis. FI $U B A$, Jul 2009, pp. 1-293.

${ }^{9}$ Barkley, D., "Linear analysis of the cylinder wake mean flow," Europhysics Letters, Vol. 75, No. 5, 2006, pp. 750-756.

${ }^{10}$ Khor, M., Sheridan, J., Thompson, M., and Hourigan, K., "Global frequency selection in the observed time-mean wakes of circular cylinders," J. Fluid Mech, Vol. 601, 2008, pp. 425-441.

${ }^{11}$ Leontini, J., Thompson, M., and Hourigan, K., "A numerical study of global frequency selection in the time-mean wake of a circular cylinder," J. Fluid Mech, Vol. 645, 2010, pp. 435-446.

${ }^{12}$ Thiria, B. and Wesfreid, J., "Stability properties of forced wakes," J. Fluid Mech, Vol. 579, 2007, pp. 137-161.

${ }^{13}$ Thiria, B. and Wesfreid, J. E., "Physics of temporal forcing in wakes," J Fluid Struct, Vol. 25, No. 4, Jun 2009, pp. 654665.

${ }^{14}$ Legendre, D., Lauga, E., and Magnaudet, J., "Influence of slip on the dynamics of two-dimensional wakes," J. Fluid Mech, Vol. 633, (2009), pp. 437-447.

${ }^{15}$ Moreau, E., "Airflow control by non-thermal plasma actuators." J. Phys. D: Appl. Phys., Vol. 39 1, 2006, pp. 262.

${ }^{16}$ E. Moreau, R. S. and Artana, G., "Electric wind produced by surface plasma actuators: a new dielectric barrier discharge based on a three-electrode geometry." J. Phys. D: Appl. Phys., Vol. 11, 2008, pp. 12pp.

${ }^{17}$ Bermudez, M. M., Sosa, R., Grondona, D., Marquez, A., Kelly, H., and Artana, G., "Study of a pseudo-empirical model approach to characterize plasma actuators," Journal of Physics Conference Series, Vol. in press, 2010.

${ }^{18}$ L.M., R. A. G., ADFC Navier-Stokes Solver, http://adfc.sourceforge.net/, 1999.

${ }^{19}$ Theofilis, V., "Advances in global linear instability analysis of nonparallel and three-dimensional flows," Prog. Aero. Sci., Vol. 39, 2003, pp. 249-315.

${ }^{20}$ González L, Theofilis V, and Gómez-Blanco R, "Finite-element numerical methods for viscous incompressible BiGlobal linear instability analysis on unstructured meshes," AIAA Journal, Vol. 45, No. 4, April 2007, pp. 840-855.

${ }^{21}$ Barkley, D. and Henderson, R. D., "Three-dimensional Floquet stability analysis of the wake of a circular cylinder," Journal of Fluid Mechanics, Vol. 322, 1996, pp. 215-241.

${ }^{22}$ Leal, L., "Vorticity transport and wake structure for bluff bodies at finite Reynolds number," Phys. Fluids A 1, 1989, pp. $124-131$. 